Meta

Journal des tradlucteurs

Translators' Journal

\title{
" Goods » et ses composés
}

\section{Jean Delisle}

Volume 16, numéro 4, décembre 1971

URI : https://id.erudit.org/iderudit/002524ar

DOI : https://doi.org/10.7202/002524ar

Aller au sommaire du numéro

Éditeur(s)

Les Presses de l'Université de Montréal

ISSN

0026-0452 (imprimé)

1492-1421 (numérique)

Découvrir la revue

Citer cet article

Delisle, J. (1971). « Goods » et ses composés. Meta, 16(4), 231-237.

https://doi.org/10.7202/002524ar d'utilisation que vous pouvez consulter en ligne.

https://apropos.erudit.org/fr/usagers/politique-dutilisation/ 


\section{“GOODS" ET SES COMPOSÉS}

Le mot goods entre dans la formation d'un grand nombre de termes dont certains présentent de réelles difficultés de traduction. L'étude qui suit vise à jeter un peu de lumière sur les problèmes que ces expressions posent au traducteur et recense les composés du mot goods les plus fréquemment usités.

Goods - Sens juridique : «Tangible movable personal property having intrinsic value usually excluding money and other choses in action but sometimes including all personal property and occasionally including vessels and even industrial crops or emblement, buildings, or other things affixed to real estate but agreed to be severed : chattels, wares, merchandise, food products, chemical compounds and agricultural products ${ }^{1}$. $\gg$ Certains auteurs précisent «not including livestock and such intangible property as leases ${ }^{2} »$. Traduction : biens corporels, biens meubles, effets.

Sens général : «Any manufactured or processed items ; any merchandise or commodities handled in commerce ${ }^{3}$. $\$$ Traduction : 1) biens : tout ce qui permet de satisfaire un besoin ; 2) produits : biens économiques qui résultent du travail de l'homme; 3) marchandises : biens qui se trouvent dans le commerce; 4) denrées : tout produit comestible par l'homme et par le bétail ; 5) matière : produit

1. Webster, 1969.

2. Ibid.

3. D. T. Clark, Dictionary of Business and Finance, New York, Thomas Y. Crowell Co., 1967, au mot goods, p. 166. 
destiné à être employé et transformé par l'activité technique; 6) production : les biens créés par l'agriculture ou l'industrie; 7) articles ; 8) objets.

Free goods : a) Biens tels que l'air, la lumière solaire, existant en quantité illimitée. Traduction : biens libres. b) (douanes) Marchandises ne payant pas de douane. Traduction : marchandises exemptes de droits, marchandises franches de droits. Ces expressions traduisent aussi duty-free goods.

Economic goods : L'existence d'un bien économique suppose trois conditions : $1^{\circ}$ un lien entre l'objet et le besoin ; $2^{\circ}$ la possibilité d'utiliser ce bien à la satisfaction d'un besoin ; $3^{\circ}$ le bien doit être relativement rare ${ }^{4}$. Traduction : biens économiques.

Les biens économiques se divisent en biens matériels (material goods) et en biens immatériels (immaterial goods, intangible goods), tels que les services, les titres et la clientèle.

On peut aussi distinguer ceux qui satisfont directement un besoin (biens directs, direct goods) ; ceux qui le satisfont indirectement, c'est-à-dire tous les produits consommés par l'industrie plutôt que par le public en général (biens indirects, indirect goods) ; et enfin, les biens mixtes (mixed goods), c'est-à-dire ceux qui servent indifféremment à la consommation et à la production d'autres biens. Aux biens directs correspondent donc les biens de consommation (consumers' goods ou consumption goods), et aux biens indirects, les biens de production (producers' goods). Un même produit peut être à la fois un bien de consommation et un bien de production, selon l'usage que l'on en fait. Le charbon, par exemple, servant au chauffage domestique est un bien de consommation ; mais s'il est utilisé pour produire de la vapeur dans une usine, il entrera alors dans la catégorie des biens de production. C'est ce qu'on appelle un bien mixte.

Consumers goods : Les biens de consommation sont ceux qui peuvent satisfaire immédiatement et directement un besoin, tels un vêtement, un jouet, un meuble, une voiture de tourisme, etc.

Capital goods, Producers' goods : «Ce sont les marchandises, le matériel, les matières, les articles qui concourent à la production, comme les outils, les machines et aussi les matières premières irremplaçables (houille, bois) qui servent de moyen de production (le blé est aussi une matière première, mais il entre dans la

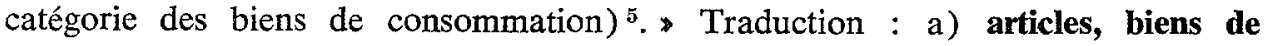
production ; b) matières premières irremplaçables ; c) marchandises-capital ; d) biens-capitaux; e) matériel d'immobilisation. «Capital goods normally excludes such items as stocks, bonds, mortgages, and money which are sometimes called representative goods ${ }^{6}$. " Cette dernière expression se rendra, selon le cas, par titre, valeur, action, valeur mobilière, obligation, billet, devise, etc. On dit aussi representative capital goods ${ }^{7}$.

De plus, il existe toute une série de termes plus ou moins synonymes de capital goods. C'est le cas de auxiliary goods (biens, matières auxiliaires), inter-

4. Grand Larousse encyclopédique, au mot bien.

5. Pierre Daviault, Langage et traduction, Ottawa, Bureau fédéral de la traduction, 1962, p. 88 .

6. Encyclopoedia Britannica.

7. Horton, Dictionary of Modern Economics, McGraw Hill, 1965. 
mediate goods (produits, matières intermédiaires), investment goods (biens d'investissement), equipment goods (biens d'équipement, biens d'investissement) et industrial goods (produits industriels).

\section{Classification selon la durée d'usage}

Les biens de consommation et les biens de production sont fréquemment classés en fonction de leur durée d'usage : non durables, semi-durables et durables.

Non-durable goods : "Products with a relatively short useful life. Sometimes, a life of six months is used as the dividing line between non-durable goods and semi-durable goods ${ }^{8}$. Exemple : aliments, tabac (biens de consommation); combustibles, produits chimiques (biens de production).

Dans son classement des industries, Statistique-Canada range les produits suivants parmi les biens non durables : aliments et boissons, tabac, caoutchouc, cuir, textiles, vêtements, papier et produits connexes, imprimés, dérivés du pétrole et du charbon, produits chimiques et produits manufacturés divers. Traduction : a) biens non durables, produits de consommation non durables; b) biens, produits consomptibles (c'est-à-dire dont on ne peut se servir sans les détruire); c) biens périssables (perishable goods); d) biens fongibles (fungible goods), soit les choses qui se consomment par l'usage et peuvent être remplacées par une chose analogue (Robert). Exemple : le blé.

Semi-durable goods : "Those products or commodities which are not immediately consumed in use, but have a short useful life. [...] Sometimes, a life of three years is considered as the dividing line between semi-durable goods and durable goods ${ }^{9}$. $\gg$ Exemple : vêtements (biens de consommation); outils (biens de production). Traduction : a) biens semi-durables; b) biens semi-consomptibles.

Durable goods : "Those products which are not normally consumed in use, and which are not further processed by their purchasers. Products with a relatively long useful life ${ }^{10}$. Exemple : meubles, appareils électroménagers, automobiles (biens de consommation); machines (biens de production).

Statistique-Canada range parmi les industries de biens durables, les industries du bois, du fer et de l'acier, du matériel de transport, des métaux non ferreux, du matériel électrique. Traduction : a) biens de consommation durables; b) biens non fongibles.

Classification par catégories de produits présentant certains traits communs

Il s'agit des fameux wet goods, dry goods, soft goods, hard goods et white goods.

Disons d'abord que ces expressions ont cours surtout dans le langage commercial et celui de la statistique industrielle, plutôt que dans le langage courant. Mais un esprit français qui veut saisir nettement ce que signifie dry goods ou wet goods se trouve un peu embarrassé : ces étiquettes ne corres-

8. Clark, op. cit., p. 167.

9. Clark, op. cit., p. 167.

10. Clark, op. cit., p. 167 
pondent pas aux catégories des classifications auxquelles il est habitué. L'anglais fait des regroupements que le français ne juge pas nécessaires. Voici ce que dit Nida à ce sujet : "Not only do languages have a distinct way of segmenting their most concrete, specific layer of existence, but they also have very different ways of distinguishing the classes in the upper layers. In fact, languages tend to be more alike on the specific concrete level and increasingly different on the higher levels ${ }^{11}$.»

Ainsi, wet goods comprend tous les produits liquides commercialisés en tonneaux, en bouteilles ou dans divers récipients. Cette catégorie regroupe donc, assez curieusement, la peinture et la bière, l'huile et les spiritueux. Toutefois, les combustibles liquides n'entrent pas dans la catégorie des wet goods. Comme traduction, on a proposé « matières liquides ${ }^{12} »$ ou 《marchandises liquides ${ }^{13} »$. Bien que ces traductions ne soient pas totalement fausses et inexactes, et qu'elles s'avèrent parfois très utiles en pratique, il n'en demeure pas moins qu'elles appartiennent à cette «langue-traduction» que l'on retrouve à la croisée de deux grandes langues qui découpent la réalité de façon différente.

Le problème de hard goods est le même. «Dans l'économie politique : nom donné aux durable consumers' goods (articles de consommation durables) comme les automobiles, les bicyclettes, les scooters, les réfrigérateurs, etc., qui, contrairement aux articles de consommation immédiate (soft goods), sont appelés à durer des années ${ }^{14} . »$ Les hard goods ne sont donc que des biens durables par opposition aux soft goods. Cette opposition procède d'un jeu de mots similaire à celui qui a donné le couple hardware et software.

Voyons maintenant le cas de soft goods et de dry goods. "In general, those consumers' goods, which are essentially textile products, such as apparel, bedding, draperies, etc., and which are usually semi-durable goods ${ }^{15}$.» Soft goods et dry goods sont synonymes. Par opposition aux wet goods (matières liquides), on a proposé comme équivalents «marchandises ou matières sèches » ou encore «denrées sèches ». Ces traductions littérales ont une résonance suspecte, et pour ma part, j'hésiterais à leur accorder leurs lettres de noblesse. Je leur préfère les équivalents mercerie, étoffes, tissus, articles de nouveauté. Le traducteur doịt toujours craindre cette menace exprimée par Voltaire dans sa dix-huitième Lettre philosophique : "Malheur aux faiseurs de traductions littérales, qui en traduisant chaque parole énervent le sens ! C'est bien là qu'on peut dire que la lettre tue, et que l'esprit vivifie.»

Le cas des white goods est plus simple. Il s'agit des gros appareils électroménagers, tels que réfrigérateurs, cuisinières, laveuses, etc., que dans le jargon de ce secteur commercial on appelle aussi big ticket appliances.

Il reste à distinguer la série impulse goods, convenience goods, shopping goods et specialty goods.

11. Eugene A. Nida, The Theory and Practice of Translation, Leiden, E. J. Brill, 1969, p. 21.

12. René Macquinghen, Dictionnaire des termes commerciaux et scientifiques, $2 \mathrm{e}$ éd., Paris, Dunod, 1960.

13. Ketteridge, Dictionary of Commercial and Financial Terms, Phrases and Practice, 1957.

14. E. et S. Deak, Grand dictionnaire des américanismes, Paris, Editions du Dauphin, 1966.

15. Clark, op. cit., p. 167. 
Impulse goods : "Those consumers' goods which a person will usually buy only if they are on display and available, rather than as the result of a definite intention to purchase ${ }^{16}$. $\gg$ Exemple : revues, friandises. Traduction : articles d'achat spontané.

L'impulse buying est un achat non réfléchi que l'on fait sous l'impulsion du moment. Achat spontané, achat par impulsion, ou parfois même achat coup de foudre rendraient bien cette idée en français ${ }^{17}$.

Convenience goods : Produits achetés périodiquement avec un minimum d'effort. Exemple : tabac, gomme à mâcher, savon, journaux, épicerie, etc., de valeur unitaire peu élevée. Traduction : a) menus articles ; b) articles d'usage ; c) articles courants.

Shopping goods : «Those consumers' goods for which a person will make a special shopping effort, rather than purchase at the most convenient source. Goods bought selectively, on the basis of price, quality and features ${ }^{18}$. "Exemple : meubles, articles de mode, etc. Traduction proposée : articles d'achat sélectif.

Specialty goods : Ce sont des articles ou des biens d'un prix généralement élevé. L'acheteur est prêt à faire un effort spécial afin de les acquérir. Ils revêtent généralement une certaine valeur (valeur esthétique, par exemple). De plus, à cause de la nature de ces biens, le consommateur peut en différer l'achat. Les objets d'art, les antiquités, les bijoux, les automobiles de luxe, entrent dans la catégorie des specialty goods. Traduction : a) articles, objets de luxe; b) biens non essentiels.

Luxury goods est un synonyme de specialty goods, catégorie dans laquelle Webster fait entrer les fancy goods (articles de fantaisie, nouveautés).

Le glossaire alphabétique qui suit donne une liste des composés du mot goods que l'on rencontre le plus fréquemment.

auxiliary goods

basic goods

big ticket appliances

bonded goods

branded goods

bulk goods

capital goods

\author{
biens, matières auxiliaires \\ matières de base \\ grands appareils électroménagers \\ marchandises d'entrepôt, \\ marchandises entreposées \\ produits de marque \\ produits en vrac \\ articles, biens de production, \\ matières premières irremplaçables, \\ marchandises-capital, biens-capitaux, \\ matériel d'immobilisation, \\ moyens de production, \\ biens d'équipement, \\ biens d'investissement \\ marchandises dédouanées \\ biens complémentaires, \\ produits à demande conjointe \\ marchandises en consignation
}

consignment goods

16. Clark, op. cit., p. 167.

17. Signalons aussi l'expression vente d'impulsion. «Vente correspondant à un acte d'achat non prévu, maís accompli au dernier moment, par exemple en effectuant un règlement à la caisse de sortie d'un livre-service " (P. Herbin, Vocabulaire de la publicité, Lagny, Editions de la Gourdine, 1964).

18. Clark, op. cit., p. 167. 
consumers' goods consumption goods contraband goods convenience goods

deadwejght goods

direct goods

dry goods

durable goods

durable consumers' goods duty-free goods economic goods equipment goods

essential goods essentials

fancy goods

fashion goods

fast-selling goods

fast-moving goods fast train goods finished goods first-rate goods foreign goods

free goods

fungible goods goods

hard goods

heavy goods high-grade goods immaterial goods impulse buying

impulse goods indirect goods industrial goods inferior goods intangible goods intermediate goods investment goods job goods light goods loose goods low-grade goods biens de consommation biens de consommation voir smuggled goods menus articles, articles d'usage, articles courants

marchandises pondéreuses, marchandises lourdes, les pondéreux

biens directs

mercerie, étoffes, tissus, articles de nouveauté

biens (de consommation) durables, biens non fongibles

voir durable goods

voir free goods (2)

biens économiques

biens d'équipement, biens d'investissement

produits de première nécessité

voir essential goods

articles de fantaisie, nouveautés

articles de mode

marchandises à écoulement rapide, marchandises de grande vente, marchandises de grand débit

voir fast-selling goods

marchandises expédiées en grande vitesse produits finis

produits de qualité supérieure

produits étrangers,

articles d'importation,

produits exotiques

1. biens libres,

2. marchandises exemptes (franches)

de droits

biens fongibles

1. biens corporels, biens meubles, effets

2. biens, produits, denrées, marchandises, matières, production, articles, objets biens (de consommation) durables voir deadweight goods

voir first-rate goods

biens immatériels

achat spontané, achat par impulsion, achat par coup de foudre articles d'achat spontané

biens indirects

produits industriels

bas produits

biens immatériels

biens intermédiaires

biens d'investissement, biens d'équipement

pacotille

marchandises légères

voir bulk goods

marchandises de qualité inférieure 


\author{
luxury goods \\ manufactured goods \\ material goods \\ measure goods \\ measurement goods \\ mixed goods \\ necessaries \\ nip goods \\ non-durable goods
}

piece goods

producers' goods

production goods

quality goods

quota goods

remote goods

representative goods

representative capital goods

returned goods

seasonable goods

second-rate goods

semi-durable goods

semi-finished goods

semi-manufactured goods

shopping goods

shop-soiled goods

shopworn goods

slow goods

smuggled goods

soft goods

specialty goods

speed goods

staple goods

style goods

weight goods

wet goods

white goods articles de luxe

produits manufacturés,

marchandises ouvrées,

produits industriels

biens matériels

marchandises de cubage,

marchandises d'encombrement

voir measure goods

biens mixtes

voir essential goods

voir style goods

biens non durables,

produits de consommation non durables,

biens consomptibles,

biens périssables,

biens fongibles

marchandises à la pièce

voir capital goods

voir capital goods

voir first-rate goods

marchandises contingentées

biens durables

titres, valeurs, actions,

valeurs mobilières, obligations,

billets, devises

voir representative goods

les rendus

produits saisonniers,

articles de saison

voir low-grade goods

biens semi-durables,

biens semi-consomptibles

produits semi-ouvrés,

produits mi-ouvrés,

produits semi-finis

produits semi-manufacturés,

produits mi-ouvrés,

produits semi-transformés

articles d'achat sélectif

articles défraîchis,

articles d'étalage

voir shop-soiled goods

marchandises expédiées en petite vitesse

marchandises de contrebande,

marchandises fraudées

voir dry goods

articles, objets de luxe, biens non essentiels

voir fast train goods

produits principaux,

principales ressources,

marchandises essentielles

articles de mode

voir heavy goods

matières liquides,

marchandises liquides

appareils électroménagers 